\title{
Hysteresis and stepwise structure in MR curves of granular superconducting ruthenocuprates $\mathrm{RuSr}_{2}\left(\mathrm{Gd}_{1.5} \mathrm{Ce}_{0.5}\right) \mathrm{Cu}_{2} \mathrm{O}_{10-\delta}$
}

\author{
B. I. Belevtsev a,*, E. Yu. Beliayev ${ }^{\mathrm{a}}$, D. G. Naugle ${ }^{\mathrm{b}}$, K. D. D. Rathnayaka ${ }^{\mathrm{b}}$, \\ ${ }^{a}$ B. Verkin Institute for Low Temperature Physics and Engineering, National Academy of \\ Sciences, Kharkov, 61103, Ukraine \\ ${ }^{b}$ Department of Physics, Texas A\&M University, College Station, TX 77843, USA
}

\begin{abstract}
Granular superconductivity effects in a polycrystalline sample of ruthenocuprate $\mathrm{RuSr}_{2}\left(\mathrm{Gd}_{1.5} \mathrm{Ce}_{0.5}\right) \mathrm{Cu}_{2} \mathrm{O}_{10-\delta}$ are studied. The main attention has been devoted to manifestation of these effects in current and magnetic-field dependences of resistive transition to superconducting state. It is found that current dependences of differential resistance taken at different temperatures intersect strictly at two definite values of current demonstrating crossing point effect. This phenomenon has been explained taking into account inhomogeneous state of intergrain medium which can be considered as a twocomponent system. The particular attention has been given to magnetoresistance (MR) hysteresis in mixed state of this inhomogeneous system and to influence of applied current and temperature on this phenomenon. Two types of hysteresis (clockwise and anticlockwise) have been found with transition from clockwise to anticlockwise hysteresis with increasing temperature. Stepwise structure in MR hysteretic curves has been observed in low-field range. Possible reasons of the change in hysteresis behavior with increasing temperature and appearance of the stepwise structure in MR curves are discussed taking into consideration inhomogeneous state of the granular superconductor studied.
\end{abstract}

Keywords: granular superconductors, ruthenocuprates, magnetoresistance

\footnotetext{
*Corresponding author. Fax: ++380-57-3403370, Phone: ++380-57-3410963

Email addresses: belevtsev@ilt.kharkov.ua (B. I. Belevtsev), naugle@physics.tamu.edu (D. G. Naugle)
} 
hysteresis, crossing-point effect

\section{Introduction}

Ruthenocuprates of composition $\mathrm{RuSr}_{2} \mathrm{R}_{2-x} \mathrm{Ce}_{x} \mathrm{Cu}_{2} \mathrm{O}_{10-\delta}$ (Ru1222), with $\mathrm{R}=\mathrm{Gd}, \mathrm{Eu}$, have attracted much attention in the last two decades as certain type of magnetic superconductors (for $0.4 \leq x \leq 0.8$ ) with $T_{c}$ up to $\approx 50 \mathrm{~K}$ $[1,2,3,3]$. Superconductivity is associated with $\mathrm{CuO}_{2}$ planes, while magnetic order is thought to be connected with the $\mathrm{RuO}_{2}$ planes. The ruthenocuprates show indications of different magnetically ordered states (from those that below $80-100 \mathrm{~K}$ are thought as being weak ferromagnetic). The nature and source of the magnetic order in these compounds is however still not clear. It has been possible so far to prepare only polycrystalline samples of Ru1222 (by a solid-state reaction method) [1, 2, 3]. The samples usually contain different impurity phases [5, 6], so that they are actually multiphase. The extrinsic disorder and inhomogeneities depend in a crucial way on the preparation conditions, especially on sintering and annealing temperature. In some studies, however, the multiphase state in ruthenocuprates is interpreted as phase separation effects [2, 7]. In any case, this makes understandable a variety of magnetic transitions reported in the literature for this system. A firm belief persists, however, that magnetic ordering is intrinsic in the primary superconducting phase in Ru1222 compounds rather than being attributed to some impurity phases.

Polycrystalline structure of the ruthenocuprates determines the pronounced granular superconductivity effects in these compounds [8, 9, 10, 11]. The results found are quite similar to those known for granular high- $T_{c}$ superconductors, and can be considered in a similar way. The system of this type can be described as an ensemble of type-II superconducting grains (with a size of a few $\mu \mathrm{m}$ ) with weak-coupling intergrain correlation. Grain boundaries represent weak links (regions of weakened superconductivity). Intergrain correlation is determined by Josephson coupling. The following types of weak links are commonly considered: (i) tunnelling of Cooper pairs across an electrically insulating boundary interface (SIS junction); (ii) overlap of superconductive wave functions of the grains in the normally conducting boundary (SNS, or proximity effect, junction); (iii) supercurrent transport through tiny superconducting constrictions ("pinholes") bridging the grains (a point contact junction); (iv) $\mathrm{SS}^{\prime} \mathrm{S}$ coupling $\left(\mathrm{S}^{\prime}\right.$ is a superconductor with lower $\mathrm{T}_{c}$ ). The 
coherence length $\xi_{G L}$ in high- $T_{c}$ superconductors is short and comparable to the grain boundary thickness. For this reason SIS Josephson junctions are thought to be of little importance in supercurrent transport while others of the above-mentioned types of the weak-coupling junctions are undoubtedly involved in current flowing in granular high- $T_{c}$ superconductors. Generally, any grain in the superconductor makes multiple contacts with neighboring grains, so that a bulk superconductor is a multiple 3D Josephson junction array [12]. The intergrain contacts of any grain can be of different types [13].

Granular superconductors have a widened (in contrast to that in homogeneous superconductors) temperature range of resistive transition to the superconducting state, with a "shouldered" form of resistive curves $R(T)$, which is also a feature of the ruthenocuprates [8, 9, 10, 11]. The shouldered form of the resistive transition reflects the inhomogeneous state of a granular superconductor. With decreasing temperature the intragrain superconducting transition takes place at temperature $T_{c 0}$, but due to poor intergrain coupling the total resistance does not go to zero. Only a small drop in resistance can be seen (if some part of the grains becomes well coupled). In other cases, a barely noticeable change in $R(T)$ curvature can be noted near $T=T_{c 0}[11]$. Intergrain Josephson coupling is strengthened with further decreasing temperature below $T_{c 0}$, so that in a complicated network of the weak links between grains some discontinuous zero-resistance paths (or clusters) appear causing further resistance drop. Due to the inevitable space distribution of grain boundary thicknesses (and, consequently, junction resistances) this resistive transition has a percolative character. As the temperature continues to fall, the superconducting clusters grow, and (if the space disorder in junction resistances is not very strong) an infinite percolating cluster is formed at a certain temperature $T_{c}$ at which the resistance becomes zero. This temperature can be far less than $T_{c 0}$.

Resistive transition to zero resistance in a weak-link network is very sensitive to applied current and magnetic field. In granular high- $T_{c}$ superconductors, magnetoresistance (MR) curves taken with increasing and decreasing external field, $H_{e x t}$, are irreversible in the temperature range between $T_{c 0}$ and $T_{c}$. It is found that this MR hysteresis has a peculiar behavior [14, 15, 16, 17, 18], which is quite different from that in homogeneous superconductors. In the latter case the resistance in the decreasing $H_{\text {ext }}$ curves is higher than that in the decreasing $H_{\text {ext }}$ ones, which is usually explained by the flux trapping. However, in granular high- $T_{c}$ superconductors the resistance when the field is decreasing is lower than that measured with increasing 
field [17, 18].

The MR hysteresis is closely connected with the magnetic hysteresis, and both of them reflect variations in the vortex state of superconducting system in the mixed state with increasing and decreasing magnetic field. Some models and explanations of specific MR hysteresis in granular high- $T_{c}$ superconductors have appeared in the first years after discovery of superconducting cuprates [14, 15, 16, 17, 18]. These have established some basic approach to consideration of this phenomenon, but due to complexity of granular systems some questions remain unanswered. For this reason rather intensive investigations of this problem continue until recent years [19, 20, 21].

MR hysteresis in the ruthenocuprates in the range of resistive superconducting transition has been noticed and discussed in [8, 9]. In this article further study of this phenomenon in the ruthenocuprates is presented. The Gd doped sample $\mathrm{RuSr}_{2} \mathrm{Gd}_{1.5} \mathrm{Ce}_{0.5} \mathrm{Cu}_{2} \mathrm{O}_{10-\delta}$ ( $\mathrm{Ru} 1222-\mathrm{Gd}$ ) has been studied. The study is to some degree a continuation of our previous studies [10, 11] of granular superconductivity effects in the Ru1222-Gd. We will describe here some new results not mentioned in previous articles [10, 11]. In particular, a stepwise structure in MR curves taken with increasing and decreasing field has been found and discussed.

\section{Samples and experimental technique}

The Ru1222-Gd samples studied have been prepared by a solid-state reaction method in I. Felner's lab [1]. Some of them were set aside (as-prepared samples), while others were annealed $\left(12 \mathrm{~h}\right.$ at $\left.845^{\circ} \mathrm{C}\right)$ in pure oxygen at different pressures. It was shown previously [11, 22] that oxygen annealing of these samples has a rather strong effect on intergrain connection but does not influence intragrain superconducting properties. This study focuses on properties of as-prepared Ru1222-Gd samples where the granularity effects were most evident. Resistance as a function of temperature and magnetic field (up to $16 \mathrm{kOe}$ ) was measured using a standard four-point probe technique in a home-made cryostat. The magnetization measurements were made with a Quantum Design SQUID magnetometer. The samples were polycrystalline with a grain size of a few $\mu \mathrm{m}$. They were characterized by resistivity, magnetization and specific heat measurements, which were in part reported in [11, 22]. 


\section{Results and discussion}

\subsection{General characterization of the sample}

Some general characteristics of transport properties the sample studied will be considered before going to the main results about MR behavior in the range of the resistive superconducting transition. Temperature dependences of the resistance $R(T)$ of the as-prepared sample $\mathrm{RuSr}_{2}\left(\mathrm{Gd}_{1.5} \mathrm{Ce}_{0.5}\right) \mathrm{Cu}_{2} \mathrm{O}_{10-\delta}$ have been recorded for different measuring currents in the range $0.01-50 \mathrm{~mA}$. Fig. 1 shows $R(T)$ dependences only for currents at which MR hysteresis was studied. The temperature $T_{c 0}=34 \mathrm{~K}$ (shown by arrow) marks a kink in the $R(T)$ dependences and is attributed to the intragrain superconducting transition. This value of $T_{c 0}$ is determined on the basis of transport, magnetic and heat capacity measurements [11,22]. Weak intergrain connection causes nonmetallic behavior of $R(T)$ above $T_{c 0}(d R / d T<0)$ with an approximately logarithmic law $(\Delta R \propto \ln T)$. Below $T_{c 0}$ resistance continues to increase with decreasing temperature but not so rapidly and only up to temperature $T_{c J}=23.2 \mathrm{~K}$ at which the maxima in $R(T)$ curves for all measuring currents takes place (Fig. 1). This presents also the branching point of the family of $R(T)$ curves taken for different currents. The temperature $T_{c J}$ indicates a temperature point below which percolating chains of intergrain Josephson coupling are created, and the resistance starts to decrease with decreasing temperature. Due to strong disorder in the intergrain coupling, the resistance in the sample studied does not go to zero with decrease in temperature down to $5 \mathrm{~K}$ (Fig. 1).

General interpretation of this $R(T)$ behavior and influence of measuring current on it can be found in [11]. As the measuring current is increased, some of the weakest links of the percolative chains with the least critical current go to the resistive state, increasing the total resistance of the system. A similar action (and for the same reasons) is produced by an applied magnetic field in the range below $T_{c J}$ (Figs. 2 and 3). It can be seen that resistance is very sensitive to magnetic field especially within the low field range. MR curves show no saturation with increasing field except for temperatures very close to $T_{c 0}=34 \mathrm{~K}$ (Fig. 3) when total superconductivity suppression is possible at rather low field.

Characterization of ruthenocuprates is incomplete without magnetization data. Most important results concerning this and similar Ru1222 samples are already presented in previous papers [11, 22]. Here we would like to show only typical magnetization hysteresis curve in the temperature range of 
superconducting state (see Fig. 4 for the Gd ruthenocuprates), which agree with known data [6]. Below $T_{c}$ the Ru1222 compounds show a ferromagneticlike behavior together with diamagnetic (Meissner) response at low applied fields [6, 11, 22]. At higher fields no saturation takes place due to paramagnetic contribution from rare-earth ions or impurity phases (Fig. 4). Changes in $M(H)$ curves with increasing temperature are shown in Fig. 5. It is seen that nonlinear low-field $M(H)$ behavior at low temperatures changes to linear at higher temperatures.

Current-voltage characteristics of the sample studied are strongly nonlinear below $T_{c J}$ but become approximately linear when approaching $T_{c J}$ (and above it). This is seen in Fig. 6 where current dependences of differential resistance $R_{d i f}=d U / d J$ are shown at different temperatures. The curves $R_{\text {dif }}(J)$ have two characteristics crossing points (the first on the ascending parts of the curves and the second on the descending part). The current dependences of dc resistance $R=U / J$ (nor shown) have the same appearance as in Fig. 6, but with higher values of the crossing point currents $J_{x 1}$ and $J_{x 2}(1.95 \mathrm{~mA}$ and $35,2 \mathrm{~mA}$, respectively). At temperatures rather far below $T_{c J}$ both, $R_{d i f}(J)$ and $R(J)$, curves show weak current dependence for small currents, but with increasing current the curves go up and then down forming the maximum (Fig. 6).

The crossing point phenomenon is well known in strongly correlated electron systems [23]. It implies that a family of curves $P(x, y)$ (where $P$ is some physical characteristic, $x$ and $y$ are some thermodynamic or dynamic quantities) can intersect strictly at one point when $P(x, y)$ is plotted as a function of one of the variable for different values of the other one. This was found, for example, for Gd ruthenocuprates in the specific-heat temperature and magnetic field curves [22]. Both crossing points in Fig. 6 have the typical appearance for this phenomenon [23]. At the same time the two crossing points observed have some clear individual features. It is seen in Fig. 6 that only curves taken at $T \leq 15 \mathrm{~K}$ show a crossing point effect. It is apparent that the first crossing point (at $J=J_{x 1}$ ) is simultaneously the inflection point for each curves intersected. This means that at this point

$$
\left.\frac{\partial^{2} R_{d i f}(J, T)}{\partial J^{2}}\right|_{J_{*}(T)}=0 .
$$

We shall try to explain the non-monotonic behavior of $R_{d i f}(J)$ curves and origins for appearance of the crossing points below. Here we would like to stress mention that the crossing point effect is a characteristic feature of 
inhomogeneous (for example, two-level, two-component, two-phase and the like) systems. It is asserted [23], among other suggestions, that the crossing point should become apparent in a system which is a superposition of two (or more) components, like that in the known Gorter-Casimir two-fluid model of superconductivity. Granular superconductors can be considered as some type of two-component or two-level system as will be discussed below.

\subsection{Hysteresis and stepwise structure in $M R$ curves}

We have studied the magnetic field dependences of the resistance and MR hysteresis for different values of the transport current (indicated in Fig.1). The $R(H)$ curves were recorded under continuous variation of the field from $-0.005 \mathrm{~T}$ to zero and then to the field $H_{\max }$ (about $1.5 \mathrm{~T}$ ) with subsequent decreasing to zero on going to the starting value of $-0.005 \mathrm{~T}$. During such cycles the same sweep rate of $20 \mathrm{mT} / \mathrm{s}$ with the field perpendicular to the transport current is kept. No significant difference in $R(H)$ curves (including the stepwise structure described below) has been found by us for measurements under other orientations of $H$ (for example, parallel or antiparallel to the current). We have found that the MR hysteresis pattern changes with increasing temperature (in the range below $T_{c J}$ ) as shown in Fig. 7. At low enough temperature, the MR curves measured with decreasing field go below those taken with increasing field (clockwise hysteresis). When temperature increases sufficiently close to $T_{c J}$ the opposite behavior takes place: the decreasing $H$ curve goes above that taken with increasing field (anticlockwise hysteresis). This change in the MR hysteresis behavior with temperature is found for the transport currents in the range $0.1-3 \mathrm{~mA}$. It is seen that under the clockwise MR hysteresis the MR curves cross in the low field range (Fig. 7 ). Similar crossing has been found in MR hysteresis of high- $T_{c}$ superconductors with weak intergrain coupling [20]. For both types of hysteresis the MR is finite ("remanent "MR) when the external magnetic field decreases to zero going to the end of the cycle. It follows from Fig. 7 and those presented below (Figs. 8, 9, and 10) that the minimum (zero) value of MR with the backward $H$ sweeping is achieved not at $H=0$ but at some negative value of $H$.

Previous studies of MR hysteresis in superconducting ruthenocuprates are scanty. Actually it is possible to compare properly the results obtained only with those in Ref. [8], where Ru1222-Gd with somewhat different composition $\left[\mathrm{RuSr}_{2}\left(\mathrm{Gd}_{1.3} \mathrm{Ce}_{0.7}\right) \mathrm{Cu}_{2} \mathrm{O}_{10-\delta}\right]$ and higher $T_{c 0} \approx 43 \mathrm{~K}$ has been studied. In that study the clockwise hysteresis at temperature rather far below $T_{c 0}$ 
and "remanent" MR are found (same as in this study). In distinction to Ref. [8] we have found the transition from the clockwise to anticlockwise MR hysteresis with increasing temperature in the range below $T_{c J}$. Moreover, we have found a stepwise structure in the $R(H)$ curves at low-field range. This structure can be seen, for example, if any curve in Fig. 7 (obtained for $J=3 \mathrm{~mA}$ ) is enlarged sufficiently, like that shown in Fig. 8. Similar stepwise structure is found to appear for other transport currents (Figs. 9 and 10). This structure appears for both, the upward and downward magnetic-field variations. It is significant that in both cases the stepwise structure appears only below some characteristic field $H_{x}$ (Figs. 9 and 10). Temperature dependences of the field $H_{x}$ together with those of $\mathrm{MR}$ at $H=1.6 \mathrm{~T}$ are shown in Fig. 11 at two currents, 0.2 and $0.5 \mathrm{~mA}$. It is evident that: (i) clear correlation exists between the temperature dependences of $H_{x}$ and MR; (ii) $H_{x}$ goes to zero with temperature going to $T_{c J}$. The latter means that the stepwise structure appears in MR curves only below $T_{c J}$.

In the range $T_{c J} \leq T<T_{c 0}$, MR hysteresis is anticlockwise and without the stepwise structure (Fig. 12). Here two main features can be observed. First, negative MR in the low-field range, and, second, saturation of resistance at high enough field when temperature is fairly close to $T_{c 0}=34 \mathrm{~K}$. Above $T_{c J}$ intergrain Josephson coupling is nearly suppressed so that intergrain conductivity is determined mainly by single-particle tunneling. In this case negative MR appears [24]. It is associated with the reduction of the intragrain superconducting gap $\Delta(T)$ in an applied magnetic field, that causes an increase in the unpaired quasi-particle density and corresponding decrease in resistance [24]. Although the intragrain upper critical field is found to be very large in the Ru1222-Gd compound [10], close enough to $T_{c 0}$, the field applied in this study (up to $1.6 \mathrm{~T}$ ) is quite enough to suppress intragrain superconductivity. It is seen in Fig. 12 that at $T=32.31 \mathrm{~K}$ the $R(H) / R(0)$ dependence shows negative MR for low field; then with further increase of the field MR becomes positive and saturates at $H>0.6 \mathrm{~T}$. Above this field the resistance is constant implying total suppression of the intragrain superconductivity by the magnetic field. MR remains positive when the field is decreased after the maximal applied field was reached (Fig. 12).

\subsection{Discussion}

The ruthenocuprates are magnetic superconductors but the internal magnetic field from the spontaneous magnetization in the Ru1222-Gd is rather 
small, below $0.004 \mathrm{~T}$ [25]. So that, in the first approximation, this will be not taken into account in our discussion.

For homogeneous superconductors the anticlockwise hysteresis of MR (when $R(H)$ under decreasing $H$ goes above that taken with increasing field) is usually explained by the flux trapping. Granular superconductors are inhomogeneous and just this determines appearance of the clockwise behavior of MR hysteresis in these materials. At present time in known literature this behavior is commonly explained on the basis of the two-level critical-state model [18] developed for high- $T_{c}$ granular superconductors. This model describes actually the two-component system which consists of (i) superconducting grains with critical current $J_{c g}$ and the lower and upper critical fields $H_{c 1 g}$ and $H_{c 2 g}$; and (ii) so called, intergrain Josephson medium with critical current and fields $J_{c J}, H_{c 1 J}$ and $H_{c 2 J}$. It is reasonably taken that $H_{c 2 g} \gg H_{c 2 J}$ and $J_{c g} \gg J_{c J}$. The field $H_{c 2 g}$ in Ru1222-Gd compounds is enormously large. For example, it was found that $T_{c 0}$ decreases by only about $2 \mathrm{~K}$ with increasing field up to $8 \mathrm{~T}$ [11]. Lower critical field of the intergranular medium, $H_{c 1 J}$, is expected to be very small (below $10^{-4} \mathrm{~T}$ according to the estimate in Ref. [16]). The intragrain lower critical field, $H_{c 1 g}$, in ruthenocuprates is poorly studied, but judging from available [3, 9] and our own data its values can be in the range $0.01-0.1 \mathrm{~T}$. It follows that even at the lowest external field the intergranular medium of the sample studied is in the mixed state (even with neglect of the inner magnetic field due to the spontaneous magnetization).

Resistivity in the mixed state of such an inhomogeneous system is determined primarily by intergrain coupling. Intergrain Josephson medium is sensitive even to small variations in current and magnetic field; whereas, these variations (at $J \leq J_{c g}$ and $H \leq H_{c 1 g}$ ) have no influence on intragrain superconducting properties. With increasing field, the flux penetrates primarily grain boundary regions with weakened superconductivity causing depression of intergrain coupling and a corresponding strong resistance rise in low field (Fig. 3). With further increasing field (above $H_{c 1 g}$ ) the flux begins to penetrate into grains. It is evident that increase in current exerts a similar influence on the intergranular medium and, thus, on measured resistance (see Fig. 1 at $J \leq J_{c g}$ ). The relevant parameter which determines behavior of resistance in magnetic field is the magnetic flux density (induction) $B_{J}$ of the intergrain Josephson medium. A clear correlation between $B_{J}$ and resistance in the mixed state of granular high- $T_{c}$ superconductors was demonstrated in Ref. [17].

It is generally accepted that based on the two-level critical-state model 
[18] the intergrain induction $B_{J}$ is determined by three contributions: (1) the applied magnetic field, $H_{a p} ;(2)$ the intragrain magnetization, $M_{g}$, and (3) the intergrain magnetization, $M_{J}$. Due to complexity of such systems, in known literature only some average expressions for $B_{J}[17,20,26,27]$ have been presented, which take into account quite generally sample shape, demagnetization fields of the sample and the grains, shape and volume fraction of grains and another factors. This can be written in a simplified form, for example, as

$$
B_{J}=H_{a p}+M_{J}\left(H_{a p}, J\right)-M_{g}\left(H_{a p}\right) C\left(H_{a p}\right),
$$

where $C\left(H_{a p}\right)$ is a shape dependent numerical factor. It is seen from Eq. (2) that $M_{J}$ and $M_{g}$ have opposite contributions to the intergranular magnetic flux density, that has been clearly shown in [26]. Due to weakened superconductivity of the intergranular medium the relation $M_{J} \ll M_{g}$ is expected to hold, so that Eq. (2) can be in some approximation rewritten as

$$
B_{J} \approx H_{a p}-M_{g}\left(H_{a p}\right) C\left(H_{a p}\right) .
$$

It is seen that hysteresis in MR in granular superconductors is determined primarily by hysteretic behavior of the intragrain magnetization, which is characterized by the intragranular flux trapping. In superconductors, the dependence of $M(H)$ with decreasing field goes above that with increasing field due to flux trapping. This leads to the clockwise hysteresis in MR in granular superconductors. The same phenomenon (flux trapping) determines the anticlockwise MR hysteresis in homogeneous superconductors since in this case the common relation $B\left(H_{a p}\right)=H_{a p}+M\left(H_{a p}\right)$ holds. In this study the transition from clockwise to anticlockwise MR hysteresis with increasing temperature has been found (Fig. 7). The anticlockwise hysteresis is fully developed when temperature is close enough to the characteristic temperature $T_{c J}=23.2 \mathrm{~K}$ above which a Josephson-like intergrain coupling is depressed although grains remain superconducting up to $T_{c 0}=34 \mathrm{~K}$. In the range $T_{c J} \leq T \leq T_{c 0}$ the anticlockwise hysteresis takes place (Fig. 12). It is evident that when $T$ approaches and exceeds $T_{c J}$ the granular system studied does not correspond to the two-level critical-state model [18] since the intergranular Josephson medium disappears and transition to one-component system takes place so that MR hysteresis becomes anticlockwise.

Now we will focus again on the stepwise structure of MR curves (Figs. 8, 9 and 10). Some stepwise structure in MR curves has been reported 
previously for a high- $T_{c}$ YBCO polycrystalline sample [19], but only very general suggestions about this phenomenon were given. Based on results of this study the following general features found for the phenomenon can be distinguished. First, the stepwise structure appears only below $T_{c J}=23.2 \mathrm{~K}$ that is in the region of the assumed two-level (or two-component) state of the granular superconductor studied. It can be suggested therefore that the jumps of resistance in magnetic field are associated with peculiarities of the flux moving in the intergranular Josephson medium. Second, the steps appear in $R(H)$ curves beginning from lowest applied fields and disappear at some characteristic field $H_{x}$, which depends on temperature and applied current and clearly correlates with MR (Fig. 11). Third, the relative amplitude of the resistance jumps or steps $\left(\Delta R / R_{0}\right.$, where $R_{0}$ is resistance in zero field) generally decreases with increasing field. This is especially evident for higher applied current (see Fig. 8 for $J=3 \mathrm{~mA}$ ). For low currents this is not so obvious (Figs. 9 and 10). The average amplitude of $\Delta R / R_{0}$ decreases with increasing current and temperature as can be seen in Fig. 13. This Figure (and Fig. 11) shows that the stepwise structure exists only below $T_{c J}$.

The stepwise structure of the MR curves suggests that penetration of magnetic field into the intergranular Josephson medium proceeds not smoothly, but discontinuously, step-by-step. This is somewhat similar (but not the same) to the phenomenon of flux jumps (or magnetic instability) known for a long time in homogeneous type-II superconductors [28, 29, 30]. In certain circumstances flux penetrates superconductors via discrete jumps or avalanches where a few or many vortices hop at once from one position to another [30]. Typically, flux jumps have revealed themselves as abrupt jumps in the magnetostriction and magnetization hysteresis loops in the Meissner state. This phenomenon in homogeneous superconductors shows itself only in samples of sufficient size (critical dimension criterion) [30]. The magnetic instability was observed in high- $T_{c}$ superconductors as well [31].

Due to the critical dimension criterion, the flux jumps in high- $T_{c}$ superconductors have been observed only in rather large single crystals or well textured polycrystalline samples with high critical current [31]. In ceramics the critical dimension is determined by grain size which is usually too small for occurrence of the flux jumps. As far as we know no such effect (like jumps in magnetization loops) was seen in polycrystalline ruthenocuprates, so that jumps in intragrain magnetization (which makes the dominant contribution to global measured magnetization) can be excluded. But below $T_{c J}$ the measured resistivity of the granular superconductor studied is deter- 
mined primarily by intergrain weak links and properties of the intergranular Josephson medium. This medium is inhomogeneous, so that its critical parameters such as $J_{c J}$ and $H_{c 2 J}$ (and, hence, intergrain Josephson coupling) have some spatial distribution. As a result of this, the increase in resistance with increasing magnetic field cannot proceed smoothly but step-by-step due to local flux jumps in the Josephson medium causing resistance jumps.

Resistance jumps in MR curves (Figs. 8, 9 and 10) can also be considered from the point of view which takes into account the percolative character of intergrain conductivity below $T_{c J}$. In a percolative granular system the conductivity is determined by the presence of "optimal" chains of grains with maximum probability of electron transport for adjacent pairs of grains forming the chain. As the applied field is increased, some of the weakest links of the chains go to a resistive state causing an increase in total resistance of the system. This process can go in steps as a discontinuous transition from less resistive to a more resistive set of "optimal" chains of grains with increasing field. It is evident that increasing field leads to a decrease in the total number of weak links at which superconducting electrons can transfer from one grain to another. The same influence is exerted by increasing temperature. In both cases, this means decreasing in area of the Josephson medium. For high enough field, the number of weak links (or volume of the Josephson area) becomes too small to produce noticeable steps in total resistance with increasing field, and the MR curves become smooth. For this reason the stepwise structure disappears above a characteristic field $H_{x}$ (Figs. 8, 9 and 10) and this field decreases with approaching to $T_{C J}$ (Fig. 11). In a resistive state of the percolative superconducting granular system even a rather low magnetic field can influence the Josephson medium and cause resistance jumps as observed in this study. This is in contrast to flux jumps in homogeneous superconductors where flux jumps are possible only above the threshold field [28].

Now we can return to discussion of the crossing point effect in the family of $R_{d i f}(J, T)$ curves (Fig. 6), taking into account that the intergranular medium which determines the total resistance below $T_{c J}$ is inhomogeneous. It consists of (1) Josephson medium which presents weak links for intergranular propagation of superconducting electrons, and (2) nonsuperconducting (normal) medium through which only transfer of unpaired quasi-particle excitations is possible. A somewhat similar concept has been applied previously for low- $T_{c}$ granular superconductors [24] where resistive transitions were explained by concurrent tunnelling of unpaired quasi-particle excitations and 
intergrain Josephson tunnelling of Cooper pairs. The intergranular medium is a typical two-component system for which, as has been mentioned above, crossing point effects can appear [23]. Relative volume of each component depends on magnetic field, current and temperature, but the total volume of the two components remains unchanged. In this case the crossing point effect is possible found in this study for $R_{d i f}(J, T)$ curves (Fig. 6). Behavior of $R_{\text {dif }}(J)$ curves is quite understandable for a two-component system. With increasing current, the volume fraction of the normal intergranular medium increases; whereas, that of the Josephson medium decreases. This leads to growth in resistance which proceeds up to some current at which maximal resistance is achieved (Fig. 6). At this current the volume fraction of the Josephson intergranular medium is zero (or close to zero). Further increase in current causes a decrease in resistance since intergranular transport of unpaired quasi-particle excitations is basically activated in character (Fig. 1) so that an increase in current enhances the probability of the intergranular transport. The nature of the second crossing point at higher current (Fig. 6 ) is unclear at the moment and deserves further study.

In conclusion, we have studied inhomogeneity effects in current and magneticfield dependences of the resistive transition to superconducting state of granular ruthenocuprate $\mathrm{RuSr}_{2}\left(\mathrm{Gd}_{1.5} \mathrm{Ce}_{0.5}\right) \mathrm{Cu}_{2} \mathrm{O}_{10-\delta}$. In the current dependence of differential resistance taken at different temperatures a crossing point effect has been found. Magnetoresistance in the mixed state of this inhomogeneous system demonstrates significant hysteresis of different type (clockwise and anticlockwise). At low temperature the clockwise hysteresis takes place; whereas, with increasing temperature the transition to anticlockwise hysteresis occurs. Stepwise structure in MR hysteretic curves has been observed in low-field range. All the mentioned phenomena can be explained by taking into consideration the peculiarities of inhomogeneous state of the granular superconductor studied, which consists of superconducting grains and inhomogeneous intergrain medium.

\section{Acknowledgements}

The authors acknowledge I. Felner (Racah Institute of Physics, The Hebrew University, Jerusalem) for providing ruthenocuprate samples. This research was supported by the Robert A. Welch Foundation (Houston, Texas) under grant A-0514. 


\section{References}

[1] I. Felner, in: A. V. Narlikar (Ed.), Studies of High Temperature Superconductors, v. 46, Nova Science, NY, 2003, pp 41-75.

[2] B. Lorenz, Y. Y. Xue, and C. W. Chu, in: A. V. Narlikar (Ed.), Studies of High Temperature Superconductors, v. 46, Nova Science, NY, 2003, pp 1-39.

[3] V. P. S. Awana, in: A. V. Narlikar (Ed.), Frontiers in magnetic Materials, Springer, Berlin, 2005, pp 531-571.

[4] P. W. Klamut, Supercond. Sci. Technol. 21 (2008) 093001.

[5] C. S. Knee, B. D. Rainford, and M. T. Weller, J. Mater. Chem. 10 (2000) 2445; V . V. Petrykin, M. Osada, M. Kakihana, Y. Tanaka, H. Yasuoka, Y. Ueki, and M. Abe, Chem. Mater. 15 (2003) 4417; J. W. Lynn, Y. Chen,Q. Huang, S. K. Goh, and G. V. M. Williams, Phys. Rev. B 76 (2007) 014519.

[6] Y. Hata, Y. Uragami, and H. Yasuoka, Physica C 468 (2008) 2392; R. Nigam, A. V. Pan, and S. X. Dou, Phys. Rev. B 77 (2008) 134509.

[7] Y. Y. Xue, B. Lorenz, D. H. Cao, and C. W. Chu, Phys. Rev. B 67 (2003) 184507.

[8] I. Felner, E. Galstyan, B. Lorenz, D. Cao, Y. S. Wang, Y. Y. Xue, and C. W. Chu, Phys. Rev. B 67 (2003) 134506.

[9] M.G. das Virgens, S. Garcia and L. Ghivelder, Eur. Phys. J. B 49 (2006) 135.

[10] V. B. Krasovitsky, B. I. Belevtsev, E. Yu. Beliayev, D. G. Naugle, K. D. D. Rathnayaka, and I. Felner, Journal of Physics: Conference Series 51 (2006) 283.

[11] B. I. Belevtsev, E. Yu. Beliayev, D. G. Naugle, K. D. D. Rathnayaka, M. P. Anatska, and I. Felner, J. Phys.: Condens. Matter 19 (2007) 036222.

[12] Thomas P. Sheahen, Introduction to High-Temperature Superconductivity, Kluwer, New York, 2002. 
[13] H. Hilgenkamp and J. Mannhart, Rev. Mod. Phys. 74 (2002) 485.

[14] Y. J. Qian, Z. M. Tang, K. Y. Chen, B. Zhou, J. W. Qiu, B. C. Miao, and Y. M. Cai, Phys. Rev. B 39 (1989) 4701.

[15] D. Dimos, P. Chaudhari, and J. Mannhart, Phys. Rev. B 41 (1990) 4038.

[16] E. B. Sonin, JETP Lett. 47 (1988) 497.

[17] Y. V. Kopelevich, Fiz. Tverd. Tela 32 (1990) 3613.

[18] L. Ji, M. S. Rzchowski, N. Anand, and M. Tinkham, Phys. Rev. B 47 (1993) 470.

[19] A. Kiliç, K. Kiliç, H. Yetiş and O. Çetin, New Journal of Physics 7 (2005) 212.

[20] D. A.Balaev, D. M. Gokhfeld, A. A. Dubrovskiy, S. I. Popkov, K. A. Shaykhutdinov, and M. I. Petrov, Zh. Eksp. Teor. Fiz. 132 (2007) 1340.

[21] V. V. Derevyanko, T. V. Sukhareva, and V. A. Finkel, Zhurn. Tekhn. Fiz. 78 (2008) 36.

[22] B. I. Belevtsev, V. B. Krasovitsky, D. G. Naugle, K. D. D. Rathnayaka, G. Agnolet, and I. Felner, J. Phys.: Condens. Matter 21 (2009) 455602.

[23] D. Vollhardt, Phys. Rev. Lett. 78 (1997) 1307; M. Eckstein, M. Kollar, D. Vollhardt, J. Low Temp. Phys. 147 (2007) 279.

[24] B. I. Belevtsev, Usp. Fiz. Nauk 160 (1990) 65 [Sov. Phys. Usp. 33 (1990) 36].

[25] G. I. Leviev, M. I. Tsindlekht, E. B. Sonin, and I. Felner, Low Temp. Phys. 33 (2007) 642.

[26] M. Mahel' and J. Pivarč, Physica C 308 (1998) 147.

[27] P. Muné, F.C. Fonseca, R. Muccillo, and R.F. Jardim, Physica C 390 (2003) 363.

[28] S. L. Wipf, Phys. Rev. 161 (1967) 404.

[29] R. G. Mints and A. L. Rachmanov, Sov. Phys. Usp. 20 (1977) 249. 
[30] Michael Tinkham, Introduction to Superconductivity, McGraw-Hill, New York, 1996.

[31] K.-H. Müller and C. Andrikidis, Phys. Rev. B 49 (1994) 1294; A. Nabialek, M. Niewczas, H.Dabkowska, A. Dabkowski, J.P. Castellan, and B.D. Gaulin, preprint arXiv:cond-mat/0209430v1 (2002). 


\section{Figure captions}

Fig. 1. (Color online) Temperature dependences of the resistance $R(T)$ of the $\mathrm{RuSr}_{2}\left(\mathrm{Gd}_{1.5} \mathrm{Ce}_{0.5}\right) \mathrm{Cu}_{2} \mathrm{O}_{10-\delta}$ sample recorded for different measuring currents. The temperature $T_{c 0}=34 \mathrm{~K}$ (shown by arrow) marks a kink in the $R(T)$ dependences and is attributed to the intragrain critical temperature of transition to the superconducting state. Another indicated temperature, $T_{c J}=23.2 \mathrm{~K}$, is discussed in the main text.

Fig. 2. (Color online) Resistive superconducting transition of the sample studied measured at current $\mathrm{J}=0.5 \mathrm{~mA}$ for different magnetic fields (in tesla): $0,0.01 ; 0.02 ; 0.03 ; 0.04 ; 0.05 ; 0.06 ; 0.07 ; 0.08 ; 0.09 ; 0.1 ; 0.2 ; 0.3 ; 0.4$; $0.5 ; 0.6 ; 0.8 ; 1.0 ; 1.2 ; 1.4 ; 1.6$. Characteristic temperature, $T_{c J}=23.2 \mathrm{~K}$, is shown by arrow.

Fig. 3. Magnetic field dependences of the magnetoresistance, $[R(H)-$ $R(0)] / R(0)=\Delta R(H) / R(0)$, of the sample studied for the temperature range $T<T_{c 0}$, recorded with increasing field at current $\mathrm{J}=0.5 \mathrm{~mA}$.

Fig. 4. Magnetization curves at $T=10 \mathrm{~K}$ of $\mathrm{RuSr}_{2}\left(\mathrm{Gd}_{1.5} \mathrm{Ce}_{0.5}\right) \mathrm{Cu}_{2} \mathrm{O}_{10-\delta}$ sample. The inset shows $M(T)$ behavior at higher field range.

Fig. 5. (Color online) Temperature evolution of magnetic field dependences of the magnetization of as-prepared $\mathrm{RuSr}_{2}\left(\mathrm{Gd}_{1.5} \mathrm{Ce}_{0.5}\right) \mathrm{Cu}_{2} \mathrm{O}_{10-\delta}$ sample in low-field range.

Fig. 6. (Color online) Current dependences of the differential resistance $R_{d i f}=d U / d J$ (obtained from current-voltage characteristics) at different

temperatures. Arrows indicate two crossing points in the set of $R_{d i f}(J)$ curves (at characteristic currents $J_{x 1}$ and $J_{x 2}$ ).

Fig. 7. (Color online) Temperature evolution of MR hysteresis curves of the sample at $J=3 \mathrm{~mA}$. It demonstrates the transition from the clockwise hysteresis at low temperature to the anticlockwise one with increasing temperature. 
Fig. 8. (Color online) Low field behavior of MR hysteresis curves of the sample at $T=8 \mathrm{~K}$ and $J=3 \mathrm{~mA}$.

Fig. 9. (Color online) MR hysteresis of the sample at $T=20 \mathrm{~K}$ and $J=0.5 \mathrm{~mA}$. Arrow indicates the characteristic field $H_{x}$ above which the stepwise structure disappears. The inset presents an enlarged image of the MR curves in the low-field region.

Fig. 10. (Color online) The same as in Figure 9 for $T=14.93 \mathrm{~K}$ and $J=0.2 \mathrm{~mA}$.

Fig. 11. (Color online) Temperature dependences of characteristic field $H_{x}$ and $\Delta R(H)=[R(H)-R(0)](H=1.6 \mathrm{~T})$ at transport currents $J=0.2$ $\mathrm{mA}$ and $J=0.5 \mathrm{~mA}$. Characteristic temperatures of granular superconductor, $T_{c J}$ and $T_{c 0}$, are indicated by arrows. The dash-dot horizontal straight line in the right corner of the upper panel presents $\Delta R(H)=0$.

Fig. 12. (Color online) Examples of MR hysteresises for $J=0.5 \mathrm{~mA}$ at temperatures close to the intragrain superconducting temperature $T_{c 0}=$ $34 \mathrm{~K}$.

Fig. 13. (Color online) Temperature dependences of the relative amplitude of the resistance jumps for different applied currents. $R_{0}$ is zero field resistance. The arrow shows the characteristic temperature, $T_{c J}=23.2 \mathrm{~K}$. 


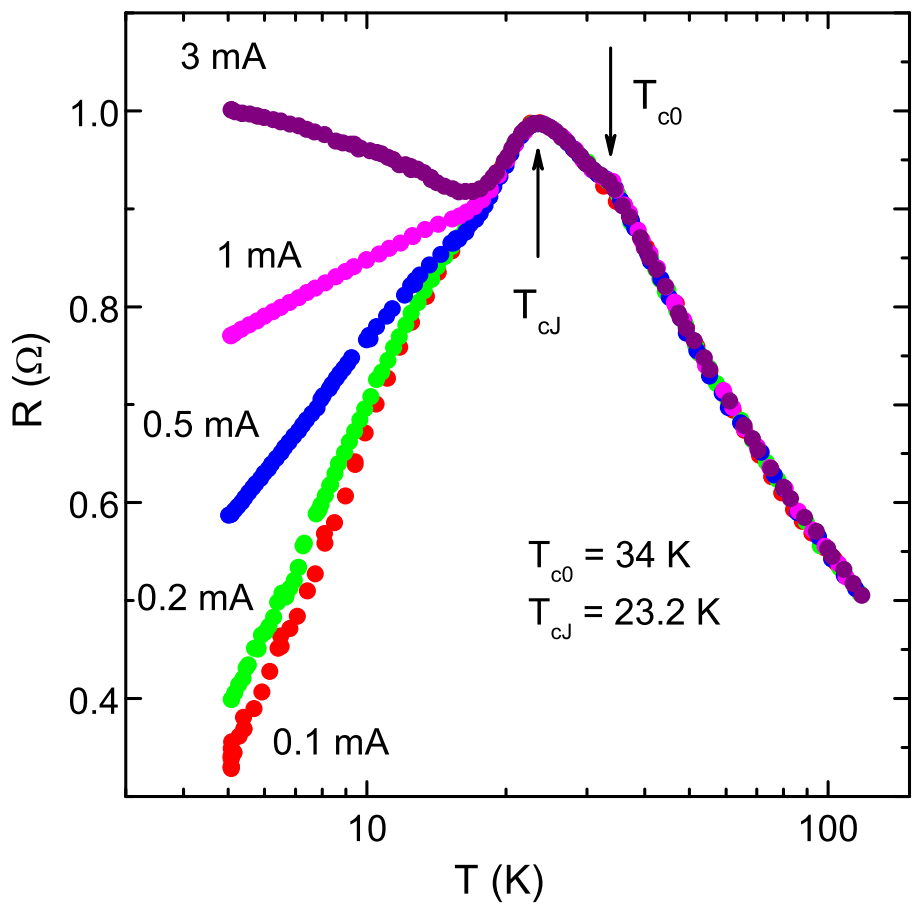

Fig. 1 to paper Belevtsev et al. 


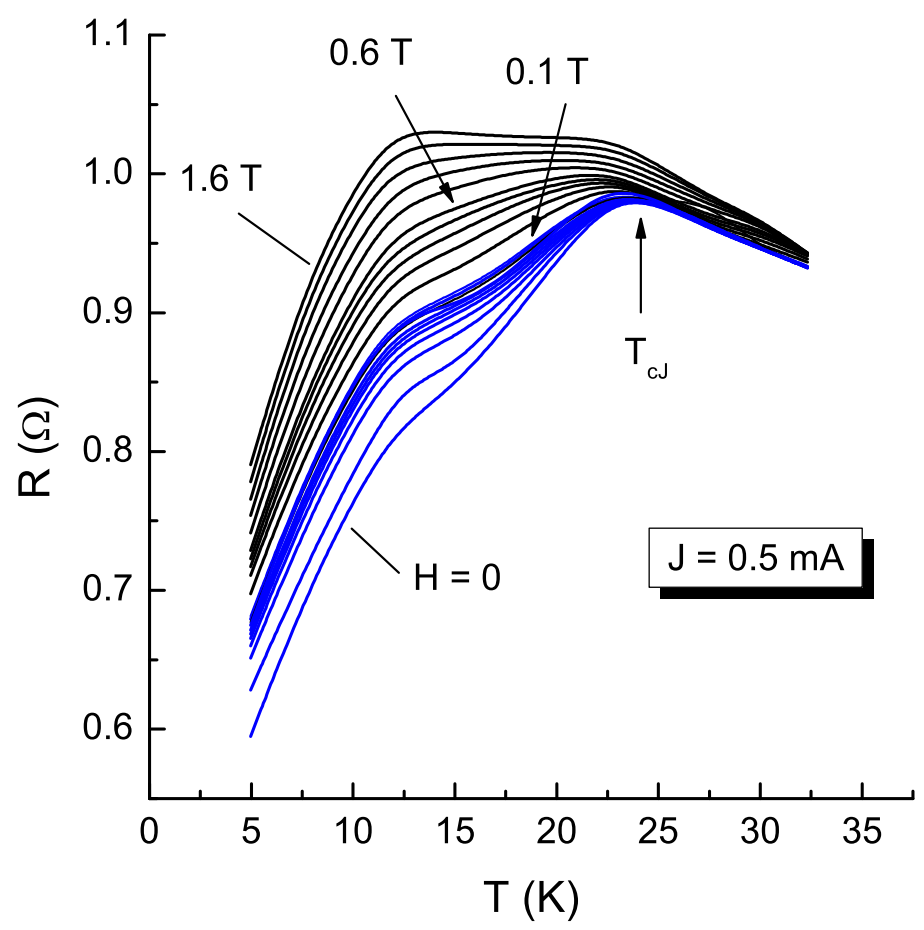

Fig. 2 to paper Belevtsev et al. 


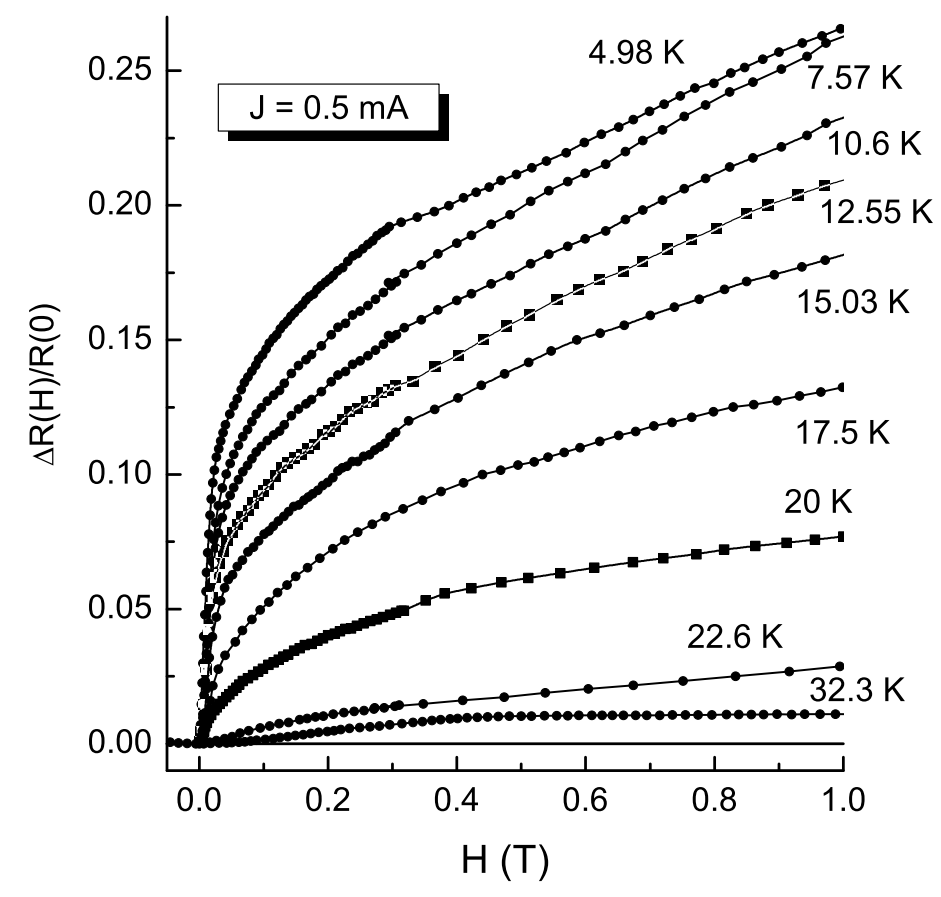

Fig. 3 to paper Belevtsev et al. 


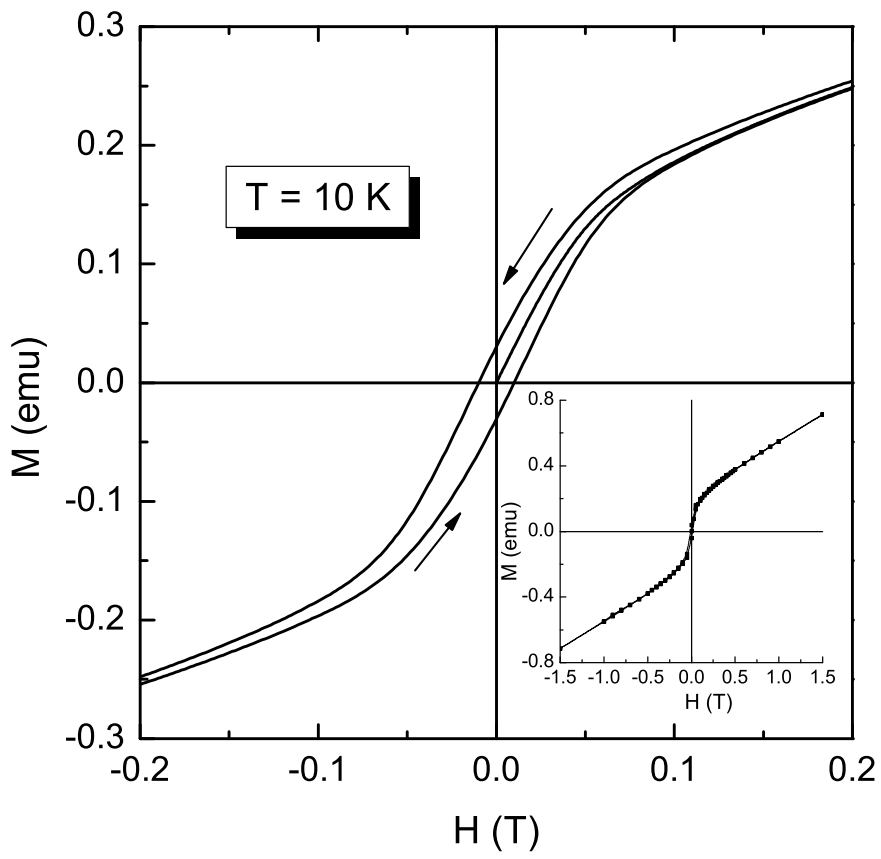

Fig. 4 to paper Belevtsev et al. 


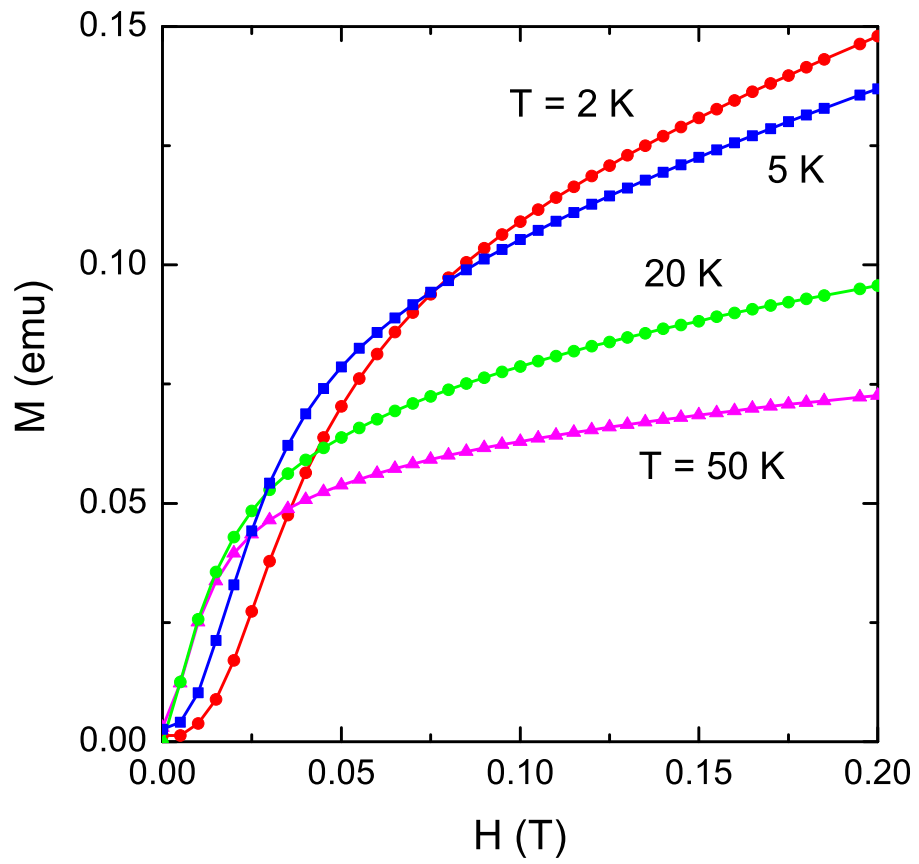

Fig. 5 to paper Belevtsev et al. 


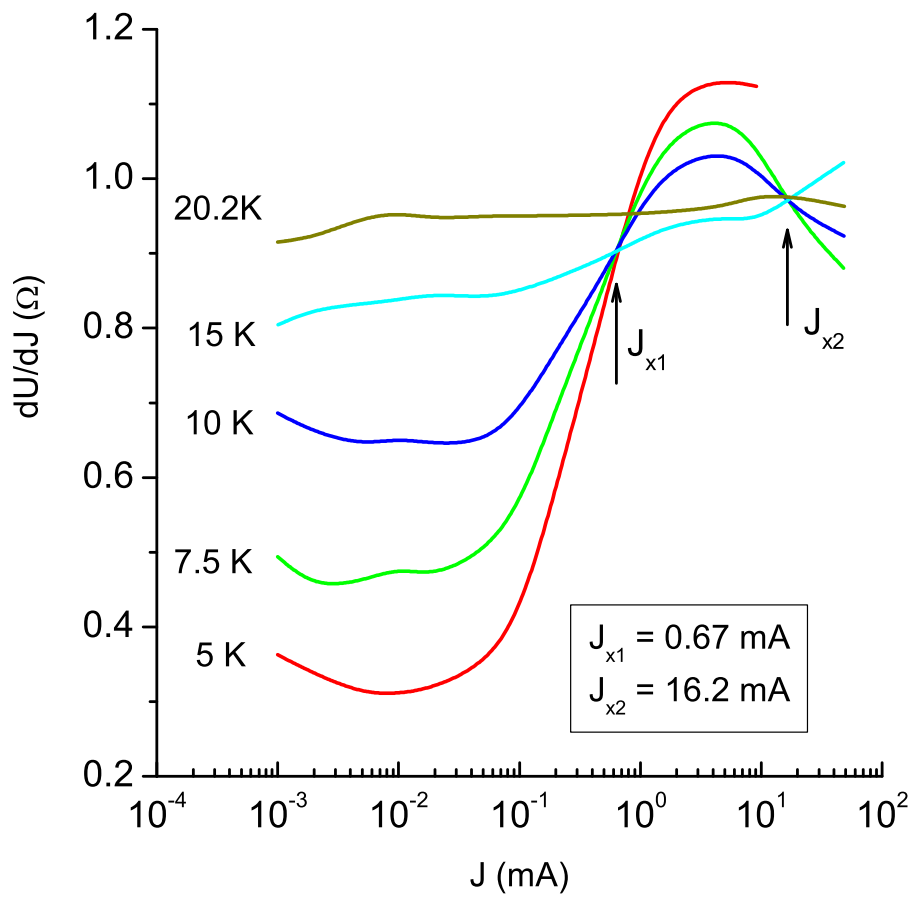

Fig. 6 to paper Belevtsev et al. 

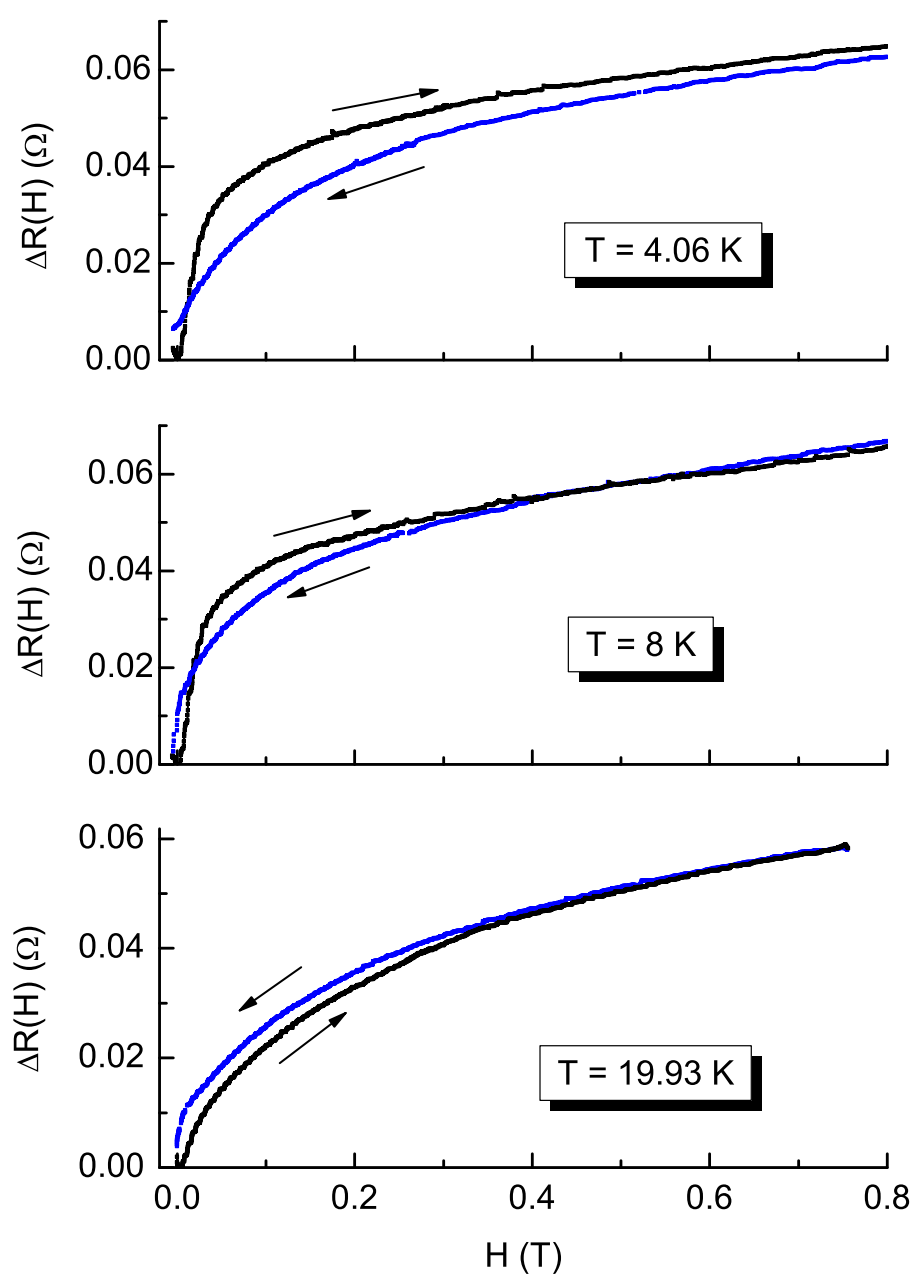

Fig. 7 to paper Belevtsev et al. 


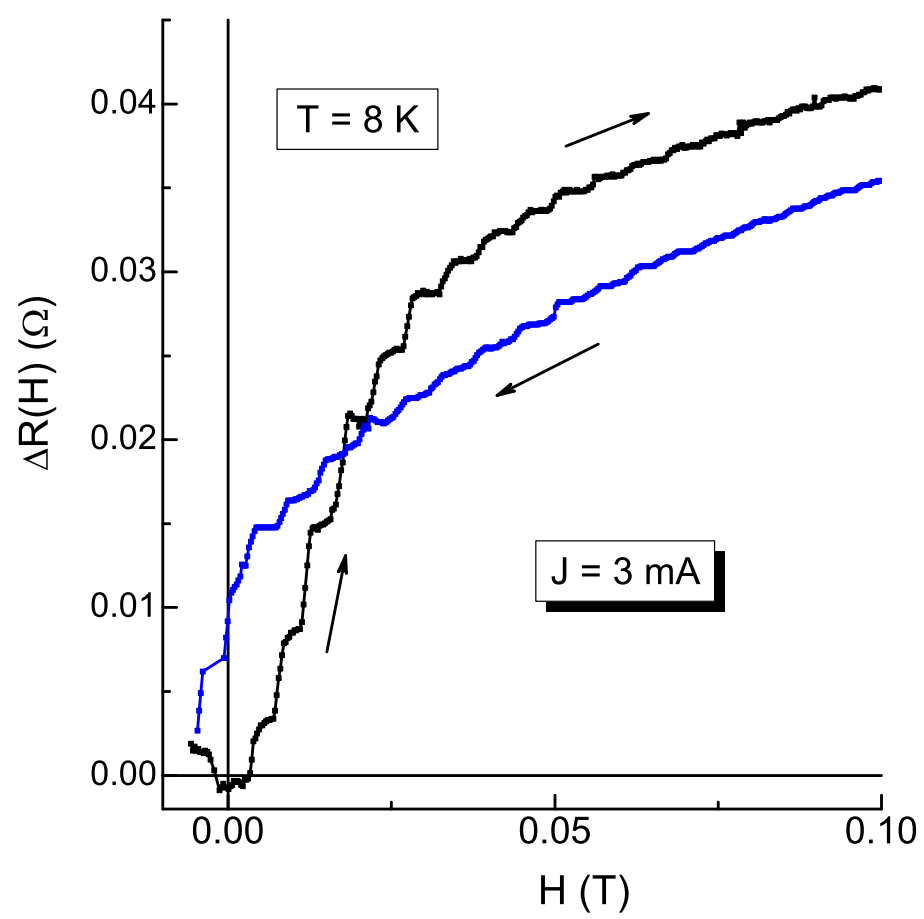

Fig. 8 to paper Belevtsev et al. 


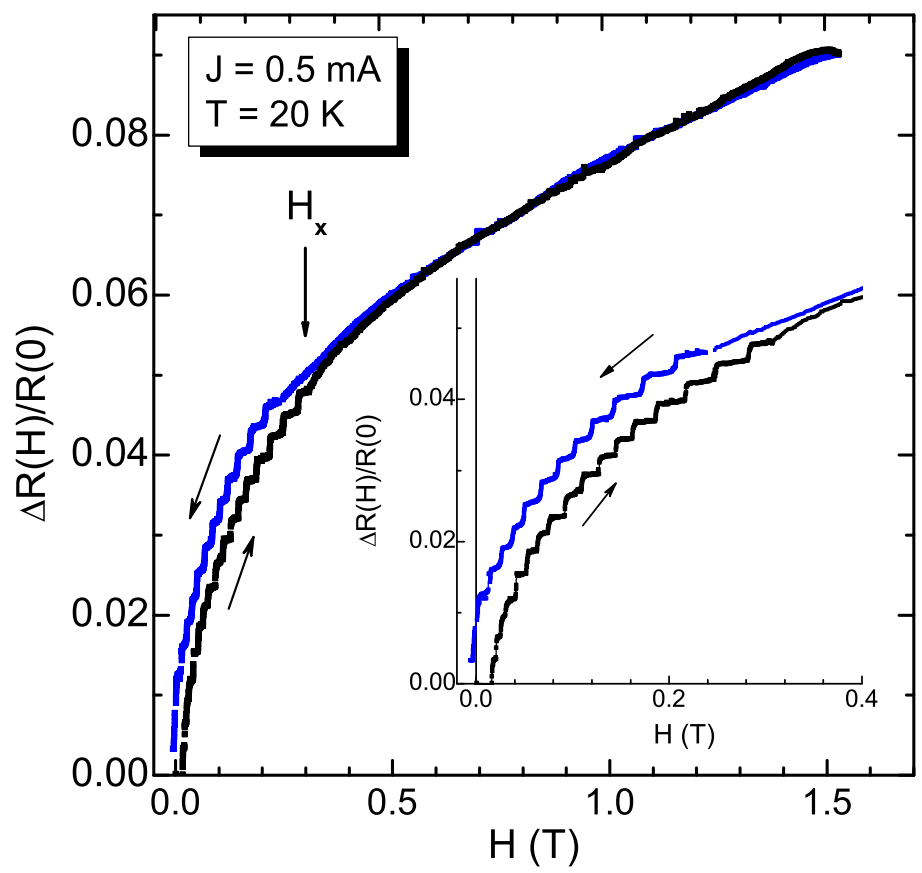

Fig. 9 to paper Belevtsev et al. 


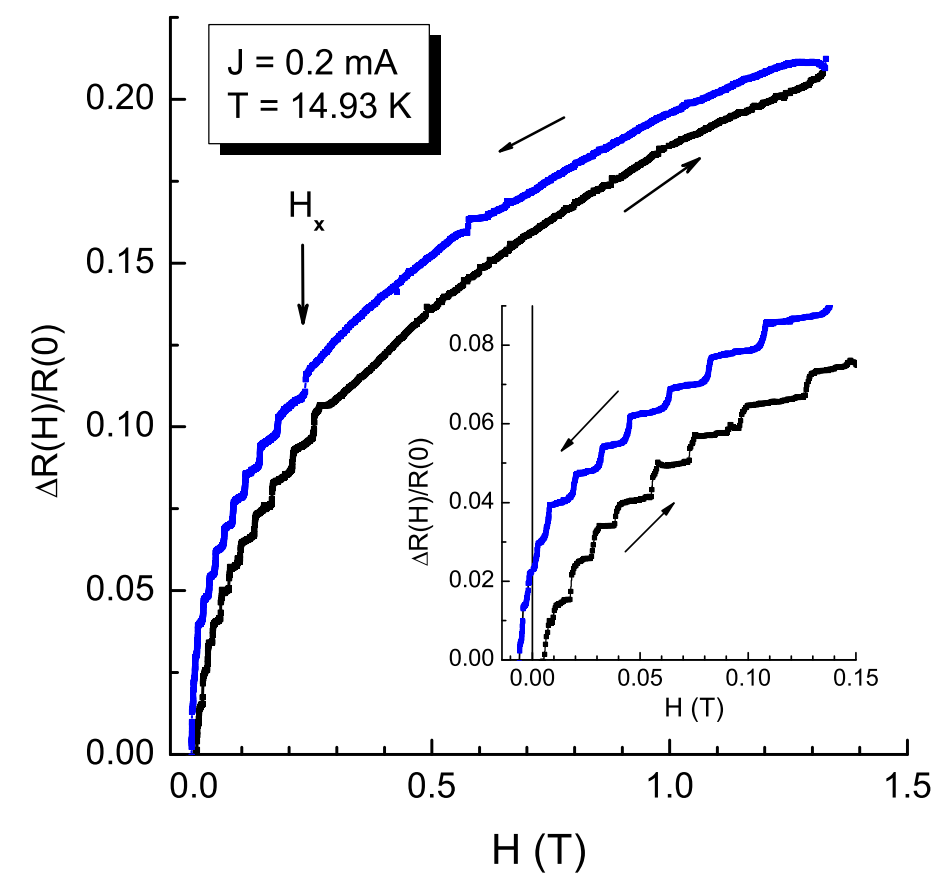

Fig. 10 to paper Belevtsev et al. 

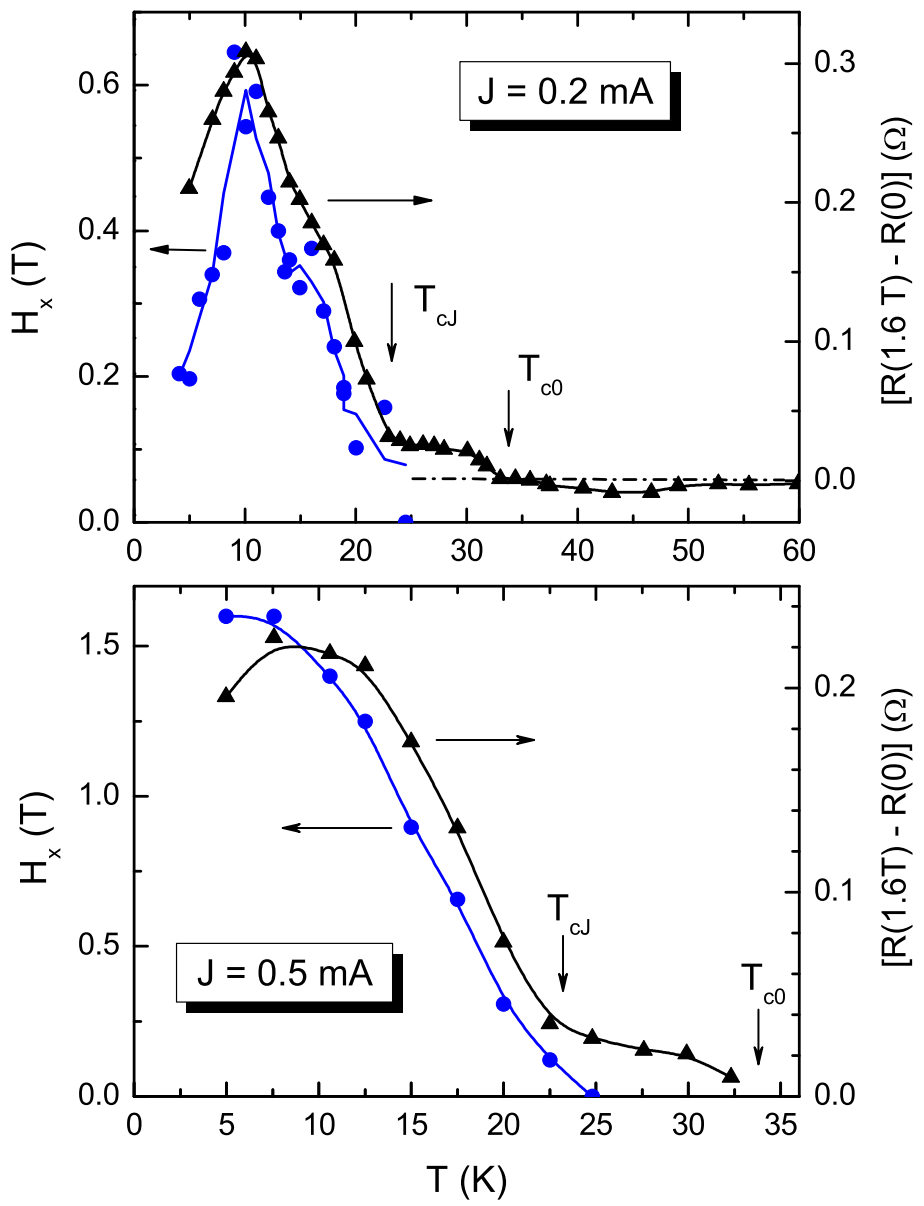

Fig. 11 to paper Belevtsev et al. 

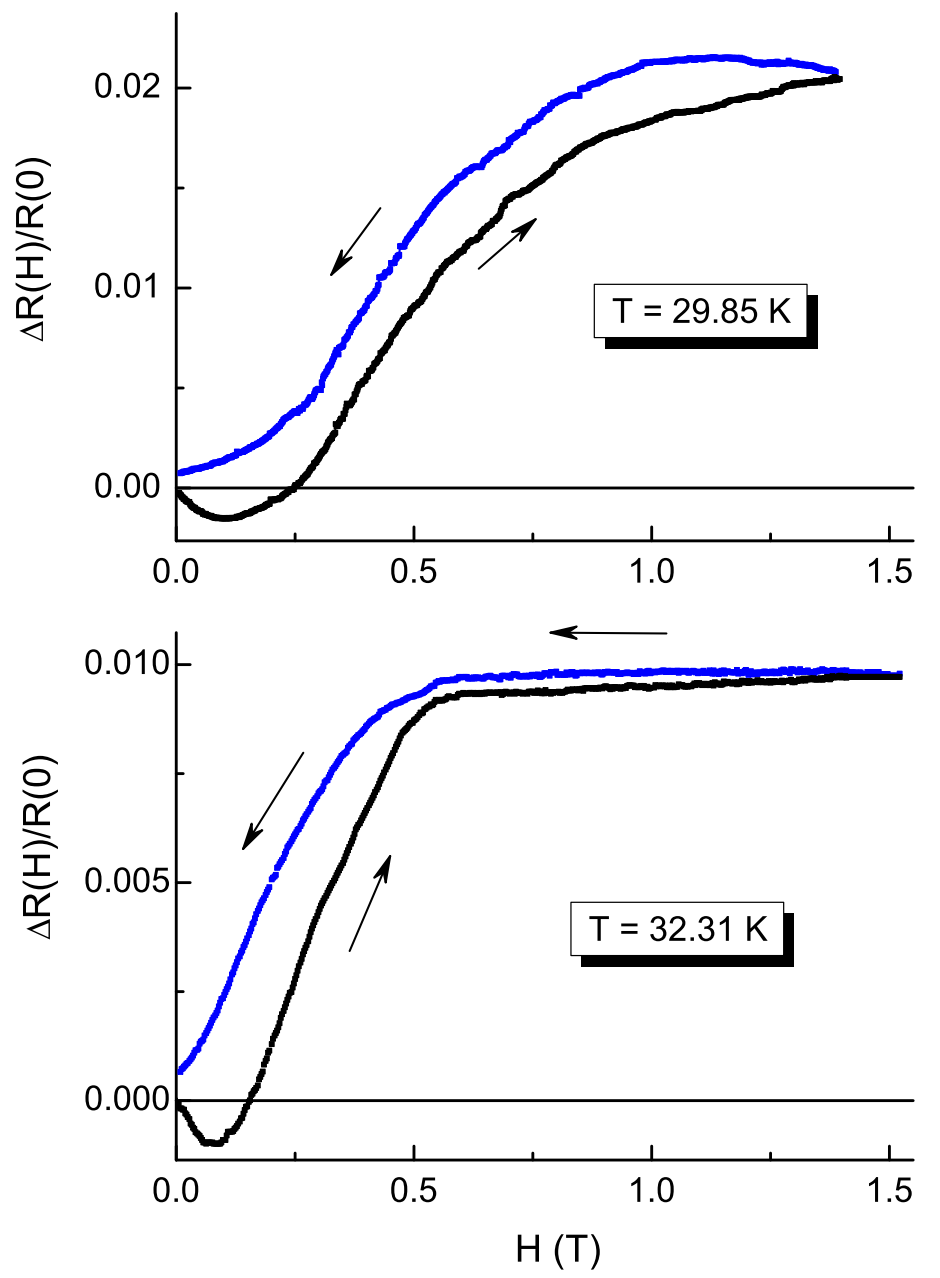

Fig. 12 to paper Belevtsev et al. 


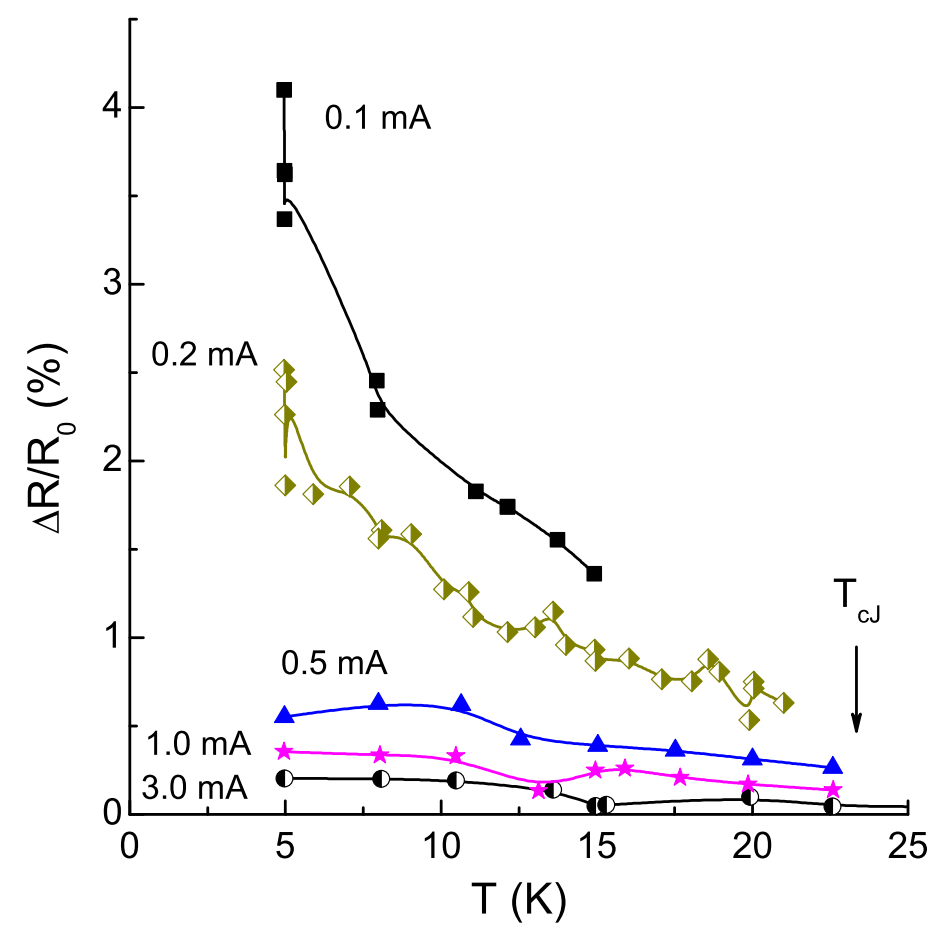

Fig. 13 to paper Belevtsev et al. 